Revue européenne des sciences sociales

European Journal of Social Sciences

\title{
Les sociologues et la crise de l'université : peur, aveuglement ou complicité ?
}

Jacques Coenen-Huther

\section{OpenEdition}

\section{Journals}

Édition électronique

URL : http://journals.openedition.org/ress/674

DOI : $10.4000 /$ ress.674

ISSN : 1663-4446

Éditeur

Librairie Droz

Édition imprimée

Date de publication : 1 décembre 2000

Pagination : 89-102

ISBN : 2-600-00481-5

ISSN : 0048-8046

Référence électronique

Jacques Coenen-Huther, « Les sociologues et la crise de l'université : peur, aveuglement ou complicité ? ", Revue européenne des sciences sociales [En ligne], XXXVIII-119|2000, mis en ligne le 15 décembre 2009, consulté le 20 avril 2019. URL : http://journals.openedition.org/ress/674 ; DOI : $10.4000 /$ ress. 674 
Jacques COENEN-HUTHER

\section{LES SOCIOLOGUES ET LA CRISE DE L'UNIVERSITÉ: PEUR, AVEUGLEMENT OU COMPLICITÉ? ${ }^{1}$}

Que l'institution universitaire soit en crise est un truisme. Qu'elle offre ainsi à l'analyse sociologique un terrain d'élection est une autre vérité d'évidence; c'est quand un système social est en mutation qu'on doit pouvoir attendre de la sociologie une œuvre d'élucidation qui confirme la pertinence de son projet intellectuel. Pourtant, face aux bouleversements qui, depuis plusieurs décennies, mettent en péril jusqu'à l'idée même d'université, la grande majorité des sociologues sont restés jusqu'à présent d'une étonnante pusillanimité. On n'oublie pas ici que des étudiants en sociologie ont joué un rôle dans les ébranlements institutionnels des années 60, mais il serait faux de dire que leur agitation fut le fruit d'une visée sociologique cohérente. Et parmi les sociologues qui réagirent immédiatement aux événements de mai 1968, Raymond Aron fut un des rares à prendre toute la mesure du danger que des idées au demeurant généreuses faisaient courir à l'institution universitaire (1968). On n'oublie pas davantage que des sociologues se sont intéressés depuis lors à l'enseignement supérieur; certains d'entre eux - et non des moindres - y ont consacré des analyses pénétrantes. On songe ici, par exemple, à l'attrait du «second marché » des intellectuels décrit par Raymond Boudon (1979) ${ }^{2}$, à l'analyse critique du monde universitaire présentée par Pierre Bourdieu (1984), aux recherches comparatives d'Erhard Friedberg $(1989,1993)$ ou aux réflexions prospectives d'Edgar Morin (1999)3. Mais le volume de travaux sociologiques consacrés à l'université, bien qu'il ne soit nullement négligeable, n'est en rien comparable à ce qu'on peut trouver dans d'autres domaines d'étude de l'action organisée: l'entreprise, l'administration publique, la vie associative, voire même l'école. Au surplus, les œuvres les plus marquantes portent davantage sur les inégalités sociales liées à l'accès à l'enseignement supérieur que sur le fonctionnement même de l'institution uni-

1 Je remercie Giovanni Busino de s'être livré à une lecture critique d'une première version de ce texte et d'avoir mis à ma disposition une documentation qui m'a permis de nuancer et de tempérer certains jugements. Il va de soi, néanmoins, que j'assume l'entière responsabilité du diagnostic présenté ici.

2 Voir aussi à ce sujet: Boudon, 1990, ainsi que mon article The Paths of Recognition: Boudon, Bourdieu and the «Second Market» of Intellectuals (1998).

3 On notera également le numéro spécial de «Sociologie du Travail» sur le thème Universités et pouvoirs (Vol. XXXI, No 4, 1989) et la Table Ronde organisée par l'Association Internationale des Sociologues de Langue Française sur L'Université en question, au cours de laquelle JeanMichel Berthelot a présenté un plaidoyer convaincant pour les processus internes d'auto-évaluation ( Université de Toulouse-Le Mirail, 1990). 
versitaire. Et surtout, alors que la majorité des sociologues sont des enseignantschercheurs dont l'université est, au moins pour une part, le milieu professionnel, on ne peut se défendre de l'impression que ce prodigieux terrain d'observation participante au quotidien ne les inspire guère ${ }^{4}$. Au sein des facultés, sur le terrain - leur terrain -, là où la crise est vécue au jour le jour, dans les salles de cours et de réunions: face aux étudiants, face aux administrateurs, face aux instances de tutelle, face aux collègues des disciplines voisines, ce ne sont généralement pas les sociologues qui dominent le jeu. Certes, comme d'autres, ils défendent les intérêts de leur unité d'enseignement et de recherche avec plus ou moins de bonheur selon les cas, mais ils semblent incapables, ou peu désireux, de présenter leur propre diagnostic de la crise ambiante ${ }^{5}$. Celle-ci les angoisse-t-elle au point de les paralyser? S'agit-il au contraire d'une cécité provoquée par une trop grande familiarité avec le milieu ou, chez les mieux établis, par une connivence de caste?

Quoi qu'il en soit, ce ne sont pas les cadres de pensée offerts par la sociologie qui structurent le débat de fond sur l'université d'aujourd'hui. Il en résulte que les enjeux sont définis par d'autres catégories d'acteurs, dont on peut raisonnablement se demander s'ils peuvent vraiment se passer des apports de la sociologie pour piloter un système aussi complexe que l'institution universitaire moderne. Que les spécialistes des sciences de l'éducation estiment avoir à cet égard une mission primordiale est tout à fait légitime. Qu'ils s'efforcent d'occuper le terrain est aussi de bonne guerre, on en conviendra aisément; ainsi en effet s'ouvre à eux un vaste champ d'activité - la pédagogie universitaire - dont ils ne pouvaient que rêver il y a un quart de siècle et qui leur offre depuis peu la perspective de s'instaurer juges de qualifications dont ils auront eux-mêmes imposé la définition. On peut craindre cependant que, livrés à eux-mêmes, ils n'en viennent à faire disparaître ce qui subsiste encore de la spécificité de l'enseignement universitaire. Que les économistes et les experts en management se sentent appelés à exercer un rôle prépondérant dans la définition des orientations de l'institution, quoi de plus normal et de plus admissible! On peut d'autant mieux le comprendre qu'une expérience de gestion fréquemment acquise dans des secteurs d'activité soumis aux exigences du marché - commerce ou industrie - leur suggère des critères d'appréciation qui peuvent paraître inattaquables. Mais, précisément, les dangers inhérents à l'extrapolation hâtive de prescriptions ayant incontestablement fait leurs preuves dans des contextes organisationnels très différents sont bien réels, d'autant plus réels qu'ils ont déjà commencé à produire leurs effets. C'est peut-être le romancier satirique britannique David Lodge qui a mis cela en lumière avec le plus de finesse persuasive dans quelques délicieux passages de son inénarrable Nice Work (1989). Si, surmontant leurs craintes ou leurs inhibitions, la majorité des sociologues cessaient de faire le gros dos et adoptaient à l'égard du milieu uni-

4 J'ai suggéré ailleurs que la pratique de la sociologie serait un exercice bien vain si les sociologues ne pouvaient tirer parti de leur savoir dans leur propre univers quotidien (Coenen-Huther, 1995a, p. 9).

5 Certains d'entre eux, parmi les plus jeunes, commencent à faire entendre leur voix, mais on est parfois étonné de les voir reprendre à leur compte un discours pédagogique oublieux des contraintes structurelles ou se résigner un peu facilement à la «secondarisation» de l'enseignement universitaire (cf. «Le Monde» du 12 mai 2000). 
versitaire la même attitude de lucidité critique dont ils font preuve ailleurs, pourraient-ils contribuer utilement au débat sur l'université? Ils pourraient, semble-til, au moins en renouveler les termes, prendre quelque recul par rapport à l'air du temps et remettre en question certaines évidences trompeuses.

La conception classique de l'université - celle qui dérive notamment du modèle de Humboldt - fait de celle-ci un lieu de création culturelle, en ce sens que la recherche et l'enseignement y sont engagés dans une dynamique de production de connaissances ${ }^{6}$. Les progrès effectués dans un contexte de découverte sont ainsi consolidés dans un contexte de présentation, ce qui exige une atmosphère intellectuelle qu'on ne doit pas craindre de qualifier résolument d'élitiste. Qui entre à l'université doit y être mû par des ambitions d'excellence; qui n'a pas de telles ambitions n'y a pas sa place. Ceci implique très nettement que la simple transmission de connaissances vulgarisées ne saurait prendre le pas sur la mission fondamentale de l'institution universitaire: promouvoir le progrès de la science ${ }^{7}$. Il en résulte également que la vocation à s'engager dans la vie universitaire n'est pas au premier chef, quoi qu'on dise et quoi qu'on fasse, une vocation à se livrer à des tâches d'enseignement mais une vocation à participer aux formes les plus hautes de la vie scientifique ou intellectuelle. La joie d'y initier de jeunes esprits est bien réelle lorsque cette activité participe d'une culture vivante du débat académique; elle ne saurait être induite par des procédures dites «d'évaluation » là où cette culture s'étiole ${ }^{8}$. Quant à la fonction de service à la cité qu'on a cru opportun de mettre en avant au cours de la décennie écoulée, il va de soi qu'elle est contenue toute entière dans la mission dévolue à l'université et qu'elle ne saurait se mesurer à l'aune de quelque application technique d'utilité locale. On peut juger cette conception de l'institution universitaire désuète et vouloir que celle-ci se transforme chaque année davantage en un lieu de passage - ou de parcage temporaire - pour des cohortes toujours plus nombreuses de jeunes gens médiocrement doués et faiblement motivés. Mais ce qu'on accepte ainsi de sacrifier est un des acquis précieux de notre civilisation occidentale. Lorsque les facultés universitaires ne seront plus que les prolongements obligés des établissements d'enseignement secondaire supérieur - qu'on les appelle collèges, gymnases ou lycées les meilleurs esprits s'en détourneront et de nouveaux centres d'excellence surgiront ailleurs. Car il n'est pas de société qui échappe à la nécessité de former des élites. On peut considérer au contraire que la conception classique de l'université mérite d'être préservée dans un contexte différent de celui qui l'a vu naître. Dans

6 Il est devenu commun d'ironiser sur le caractère daté de ce modèle, mais les essais de séparation institutionnelle de l'enseignement supérieur et de la recherche ne se sont guère révélés concluants jusqu'ici, comme en témoigne l'expérience française du CNRS vieillissant. Quelle que soit la part d'idéalisation nostalgique que peut comporter la conception libérale d'une communauté vouée à la vie intellectuelle et à la science - idéalisation déjà signalée par Max Weber au début du XX siècle - elle garde la force d'un mythe fondateur irremplaçable (Voir à ce sujet: Walter Rüegg, 1997).

7 C'est bien ainsi que l'envisageait Wilhelm von Humboldt dans son Antrag auf Errichtung der Universität Berlin en 1809, comme nous le rappelle fort opportunément Günter Endruweit (1997).

8 Et comme le fait observer notre collègue Richard Münch, de l'Université de Bamberg, «Um das Fortbestehen der Akademischen Reflexionskultur... muss man sich heute allerdings Sorgen machen» (1998, p. 150). 
cette optique, chaque proposition de réforme doit alors être scrutée soigneusement afin d'en déceler à temps les effets pervers éventuels. C'est en revanche manquer singulièrement de clairvoyance que de rendre rituellement hommage au modèle d'université que nous a légué le XIX ${ }^{\mathrm{e}}$ siècle tout en favorisant systématiquement des mesures qui ne peuvent qu'en saper les bases et faire de l'université moderne une haute école peu distincte des autres ${ }^{9}$. Sur ce point, le message de la sociologie est que tout système social comporte des compatibilités et des incompatibilités, que tout n'est pas simultanément possible et que l'attitude la plus malencontreuse est celle qui n'est pas cohérente avec les choix proclamés.

Revendiquer pour l'université le droit d'être fidèle à sa mission en restant un foyer de culture et un centre d'excellence exige en effet des options courageuses. On heurte ici de front des conceptions naïvement égalitaires selon lesquelles tout un chacun aurait le droit d'entrer à l'université et d'en sortir muni d'un diplôme. En Suisse comme dans d'autres pays d'Europe occidentale, l'autorité politique impose l'accès direct aux facultés universitaires pour toute personne ayant acquis un diplôme de l'enseignement secondaire. L'idée de sélection à l'entrée suscite de telles réactions d'incompréhension ou d'indignation que rares sont les responsables politiques qui se hasardent à affronter l'opinion sur ce point. Les mécanismes de sélection qu'on n'ose pas mettre ouvertement en place sont remplacés vaille que vaille par une sélection de facto en première année d'université. Les taux d'échecs à ce stade varient selon les pays et selon les facultés mais il est fréquent qu'ils dépassent la moitié de l'effectif initial, donnant lieu à des redoublements, à des réorientations ou a des abandons purs et simples. Le rôle du sociologue est de faire observer en l'occurrence qu'il y a là un choix de société exagérément coûteux pour la collectivité, dont il est par ailleurs parfaitement saugrenu de faire porter la responsabilité aux facultés concernées, comme on tente régulièrement de le faire. Il appartient au sociologue de mettre en évidence l'énorme gaspillage d'énergies et de ressources qu'entraîne ce refus persistant d'affronter un égalitarisme irréaliste et non de spéculer peu dignement sur le gonflement d'effectifs estudiantins qui masquent mal un désastre éducatif ${ }^{10}$. Nos sociétés restent, il est vrai, profondément inégalitaires. Aux inégalités traditionnelles qui n'ont pas disparu viennent s'ajouter des inégalités nouvelles (Fitoussi et Rosanvallon, 1996). Mais l'université n'est certainement pas le lieu d'où l'on peut prétendre y porter remède. A vouloir à tout prix assigner à une institution une fonction qu'elle ne peut assumer, on accumule inévitablement les effets pervers de tous ordres. «Jaurès voulait que les enfants du peuple reçoivent une culture équivalente à celle de la bourgeoisie » rappelle Alain Finkielkraut qui poursuit: «Les parents instruits et avisés de la bourgeoisie rêvent aujourd'hui que leurs enfants bénéficient d'une culture équivalente à celle qu'ils ont reçue et ils sont prêts à y mettre le prix. Ils usent de tous les stratagèmes, de tous les subterfuges et de tous les déménagements pour trouver une école primaire, puis un collège, puis un lycée

Soit dit en passant, c'est précisément ce que fait en Suisse le rapport Pour l'Université signé par Charles Kleiber, Secrétaire d'Etat à la Science et à la Recherche, et préfacé par la Présidente de la Confédération ainsi que par un membre du Conseil Fédéral.

10 Il ne s'agit évidemment pas de réduire l'accès à l'enseignement supérieur mais d'aller résolument de l'avant dans la voie de sa diversification. 
- privé ou public - où la communication n'a pas détrôné la transmission, où l'émulation n'est pas taboue, où l'idée de mérite est considérée comme un acquis démocratique et non comme un scandale pour la démocratie, où l'on ne s'adosse pas à la misère pour faire honte à la pensée » (2000, p. 19). Il n'est guère besoin de beaucoup d'imagination pour transposer ce scénario dans l'enseignement supérieur des prochaines décennies.

Dans une conjoncture marquée tout à la fois par la massification de l'enseignement et par les restrictions budgétaires, la réaction bien compréhensible des responsables politiques et administratifs est de chercher à se doter de normes d'allocation des ressources matérielles et humaines réduisant au maximum la part d'arbitraire dans les prises de décision. Dès lors, la tentation est grande d'escamoter le rôle essentiel d'instance socialisatrice de l'institution universitaire et de l'assimiler à n'importe quel organe d'offre de services afin de pouvoir lui appliquer des critères d'appréciation irréfutables. Les gestionnaires de l'enseignement et de la recherche se mettent ainsi en devoir de définir des «indicateurs de performance » permettant d'établir un «contrôle de qualité » aussi objectif que possible. S'agissant de l'enseignement, on en vient à émettre des jugements lourds de conséquences pour l'avenir des institutions concernées sur la base de statistiques portant sur les échecs, les abandons, la durée moyenne des études, le nombre de diplômes décernés ou de thèses soutenues avec succès dans des délais relativement brefs. Ne serait-ce pas le rôle des sociologues de clamer sans ambages qu'aucun de ces indices prétendument objectifs n'est exempt d'ambiguïté? Qu'il n'est rien de plus aisé que de provoquer une amélioration apparente de la «performance» d'un établissement d'enseignement supérieur en abaissant le niveau des exigences à l'égard des étudiants! Que dans la conjoncture actuelle, il faut au contraire un courage civique confinant à l'héroïsme pour ne pas céder à cette tentation si payante à court terme ! ${ }^{11}$ Que la majorité des étudiants de premier et de deuxième cycle ne sont pas ces jeunes adultes avisés, à la recherche de la meilleure formation possible, évoqués par le rapport Kleiber (p. 34), mais bien des adolescents prolongés, avides de gratifications immédiates, considérant l'enseignement universitaire comme une vaste tombola de crédits d'études (Zijderveld, 1991, pp. 89-90) et prêts à nouer avec le corps enseignant des rapports de complicité tacite fondés respectivement sur la complaisance et l'indolence!

Sous-jacent aux prétendus «indicateurs de performance» qui, en milieu universitaire, ne peuvent que tendre à se transformer en indicateurs d'opportunisme, il y a un modèle simple d'input-output dont on comprend qu'il soit de nature à séduire pas mal de gestionnaires. L'université accueille des cohortes successives de jeunes gens, leur offre des prestations qu'il y a lieu d'évaluer en termes de rendement et de valeur ajoutée, et le résultat de ces efforts doit pouvoir s'apprécier en fonction du temps nécessaire aux jeunes diplômés pour accéder au marché de l'emploi, à un niveau correspondant à ce que la formation reçue leur permet

11 Tentation à laquelle nombre d'enseignants ont déjà cédé aux Etats-Unis, allant bien au-delà de la mansuétude excessive: «At a time when superintendents are under pressure to increase test scores and hold principals and teachers accountable for student success, talk of cheating dominates the conversation in education circles.» (Mathews and Argetsinger, International Herald Tribune, June 3-4, 2000). 
d'espérer ${ }^{12}$. Bien entendu, ce mode de raisonnement se heurte à une objection fondamentale: les institutions éducatives en général, et l'université en particulier, constituent des organisations d'un type particulier, face auxquelles le schéma classique d'input-output perd toute valeur opératoire. Tout d'abord, la complexité de la relation enseignant-enseigné interdit toute comparaison facile avec l'offre d'un produit à une clientèle. Cela, les plus clairvoyants des économistes l'ont compris depuis longtemps (Bienaymé, 1986, p. 29). Ensuite, cette analogie même, à supposer qu'elle ait un sens, masque une irréductible «ambiguiité des préférences ${ }^{13}$. Comme j'ai eu l'occasion de le noter ailleurs, le statut d'étudiant universitaire correspond à une phase de la vie marquée simultanément par l'élargissement du champ des possibles, par une relation ambiguë à l'autorité, parentale ou autre, par une abondance de sollicitations nouvelles, tant affectives que cognitives, ainsi que par une restructuration des formes de sociabilité (Coenen-Huther, 1989, p. 778). L'anticipation naïve d'un rapport rationnel aux études ne peut que buter sur l'entrecroisement des logiques de comportement et l'importance de la composante affective latente dans les choix effectués (ibid., p. 779). Davantage encore que les comportements d'adultes engagés dans la vie active, les comportements estudiantins ne peuvent s'analyser que dans une optique de rationalité limitée (March et Simon, 1958, 1964). Il est en effet illusoire de les traiter selon le schéma classique de l'optimisation de variables. Bien au contraire, il y a le plus souvent arrêt sur la première solution jugée satisfaisante selon les critères du moment, avec réorganisation fréquente du cadre cognitif dans lequel s'insèrent ces critères (CoenenHuther, 1989, pp. 779-780). Il en résulte des contextes de décision où « les acteurs... ne savent pas très bien ce qu'ils veulent, ou plutôt ils veulent beaucoup de choses pas toujours faciles à concilier» (Friedberg, 1993, pp. 70-71). Mais la valeur opératoire du schéma d'input-output se heurte également au caractère difficilement pénétrable du processus de transformation de l'input. Les techniques mises en œuvre résistent précisément à l'examen critique sur base de critères simples. Les inputs et les outputs du système ne sont que très malaisément définissables de manière univoque. La séquence intermédiaire est plus opaque encore car tout système éducatif est un système ouvert dont la frontière avec l'environnement ne peut être tracée qu'avec une part d'arbitraire. Divers agents socialisateurs formels et informels sont en compétition permanente. Il n'est guère sérieux de prétendre évaluer la part des enseignements reçus dans la somme des connaissances d'un individu; il est moins sérieux encore de s'en remettre à cet effet au jugement subjectif de l'intéressé lui-même. Il n'est pas davantage possible de supputer l'influence réelle de tel ou tel aménagement des programmes sur les processus d'apprentissage $^{14}$. Nous sommes ici dans le domaine des paris plus ou moins bien intention-

12 Je ne cultive pas le sarcasme à plaisir; je me borne à paraphraser des documents relatifs à une évaluation de départements universitaires organisée par le Vlaamse Interuniversitaire Raad [Conseil Interuniversitaire Flamand], à laquelle j'ai participé et dont les principes s'inspiraient d'une procédure conçue aux Pays-Bas.

13 Pour s'exprimer, à la manière de Friedberg (1993, p. 71), à la suite des économistes et des théoriciens de la prise de décision.

14 Contrairement à ce qu'imaginent, à d'autres niveaux, les apprentis-sorciers des réformes scolaires auxquels sont livrés ceux qui n'ont pas les ressources de la bourgeoisie: «les autres... ceux qui font confiance, ceux qui n'ont pas le bras assez long, les mal lotis et les mal nés...» (Finkielkraut, 2000). 
nés, débouchant très fréquemment sur des conséquences inattendues. A cet égard, comme le fait observer Friedberg, toutes les argumentations sont dès lors également recevables (1993, p. 71). L'ambiguïté des préférences, jointe à l'opacité des processus de transformation de l'input, suscite une grande part d'imprévisibilité dans les procédures de prise de décisions, qu'il s'agisse de leur objet, de la définition des priorités ou de la participation des acteurs. De telles organisations ont été qualifiées de loosely coupled systems ${ }^{15}$ (Weick, 1976) caractérisés «by the ambiguity of goals, by the lack of clarity in technology, and by the transient character of many participants » (Cohen et al., 1976, p. 25). Les prises de décision effectives y relèvent du garbage can decision process en ce sens qu'elles résultent de la conjonction d'ordres de préoccupations relativement hétérogènes (ibid., p. 26). On a voulu y voir un état d'anomie, en se référant aux formes anormales de la solidarité organique évoquées par Durkheim et ceci reflète certainement un état de fragmentation de l'activité scientifique qu'on peut déplorer (Musselin, 1990, p. 458). Mais il est important de bien comprendre qu'il s'agit de caractéristiques intrinsèques des systèmes éducatifs, liées à des contraintes structurelles internes et externes, et non de dysfonctions pouvant être éliminées par l'action volontariste de quelque gestionnaire à poigne. Dès lors, ne conviendrait-il pas que ceux qui, parmi les sociologues, ont l'énorme privilège de bénéficier personnellement d'une assise institutionnelle stable et n'ont rien d'autre à craindre que l'impopularité se décident à proclamer que les catégories conceptuelles du marketing - nos produits, nos prestations, nos clients - ne sont décidément pas de mise à l'université?

L'institution universitaire est submergée par des vagues successives de jeunes gens peu préparés à y étudier avec fruit. Faute d'autres solutions politiquement acceptables, ceux qui président à son destin tendent à adopter une attitude qu'on ne peut s'empêcher de comparer à ce qui s'observe en matière de régulation du trafic automobile: songer en priorité à élargir les routes et multiplier les places de parc à mesure que les voitures se font plus envahissantes. Mais il n'est pas d'autoroute à six pistes qui offre un accès sans effort aux fruits de l'activité scientifique et aux récompenses de la vie intellectuelle. Toutes les mesures inspirées démagogiquement, il faut bien le dire - de cette logique d'élargissement de l'accès ne peuvent que manquer leur effet ${ }^{16}$. Dans le contexte général de conceptions éducatives qui ont favorisé l'éclosion de la «culture du narcissisme ${ }^{17}$, un discours suggérant aux étudiants qu'ils sont les clients de l'université les transforme effectivement en clients d'autant plus exigeants qu'ils sont moins lucides quant à leurs propres limites. Les parcours universitaires «à la carte» se multiplient au point de dépasser les capacités de gestion administrative des programmes d'études en cause. Le droit à entreprendre des études supérieures se transforme inexorablement en droit à les terminer avec succès ${ }^{18}$. Le droit à s'exprimer de

15 Expression de loin préférable à celle d' «anarchie organisée » utilisée dans la littérature de langue française, qui comporte une connotation négative tout à fait injustifiée.

16 Ou pire encore: introduire en milieu universitaire des phénomènes d'incivilité dont l'université aurait dû pouvoir faire l'économie (cf. Lepoutre, 2000).

17 Je fais allusion ici, bien entendu, à l'ouvrage de Christopher Lasch qui déplore «a new erosion of standards in the name of pedagogical creativity» (1979, p. 144).

18 Dans certaines facultés, une situation d'échec fait presque à coup sûr l'objet d'un recours auprès de l'autorité académique concernée. 
façon irréfléchie l'emporte sur le devoir d'écouter et de s'instruire préalablement; on feint même d'y voir le principe d'innovations pédagogiques louables. Et surtout, à une époque où les jeunes mûrissent de plus en plus tardivement, on affecte de les faire participer de plus en plus tôt à des prises de décision dont les enjeux dépassent largement leurs facultés d'appréciation. Ce faisant, on oublie que toute relation de professeur à élève comporte nécessairement un aspect directif et que les jeunes ont moins besoin de parodie de démocratie que de maîtres qu'ils puissent respecter. Les sociologues en poste à l'université, pourtant bien entraînés à repérer les effets pervers des décisions partout où ils se manifestent, ne s'expriment à ce sujet qu'en privé. Ne serait-il pas temps qu'ils attirent fortement l'attention sur la délégitimation accentuée de la fonction d'enseignant qui est en train de s'opérer ainsi et qui va exactement à l'encontre de ce qu'on prétend promouvoir: davantage de considération pour les tâches d'enseignement!

Force est bien de le constater en effet: dans les circonstances actuelles, le souci de rester fidèle à sa vocation d'universitaire passe précisément par une prise de distance mentale à l'égard de la population estudiantine ${ }^{19}$ ! Un universitaire de talent ne peut pas se permettre de laisser les étudiants devenir pour lui des «autres significatifs » (significant others), c'est-à-dire, dans la tradition de la sociologie interactionniste s'inspirant de George Herbert Mead, des individus dont les jugements pourraient avoir prise sur ses propres états d'esprit. C'est pourtant bien ce que visent à produire les procédures d'évaluation des enseignements dont on tend à multiplier la fréquence ${ }^{20}$. On ne songe nullement à contester ici que les questionnaires distribués aux étudiants - établis avec compétence et utilisés avec discernement - puissent fournir des indications utiles dans le cadre d'un dialogue institutionnalisé entre partenaires voués aux mêmes valeurs. En revanche, mis à la disposition de jeunes gens dont il n'est pas exagéré de dire avec feu Allan Bloom qu'ils sont souvent dépourvus de tout intérêt intellectuel, «ignorant même qu'il existe quoi que ce soit qui réponde à cette définition» $(1987, \text { p. 282 })^{21}$, ils ne peuvent que se transformer en redoutables instruments de chantage moral et favoriser le laxisme éducatif, la recherche d'une popularité facile, les attitudes démagogiques; car on ne saurait fonder un enseignement de qualité sur des sondages d'opinion $^{22}$. Il appartient aux sociologues de dévoiler tout d'abord l'enjeu politique et institutionnel que dissimule la promotion des «aptitudes pédagogiques». Celle-ci permet tout d'abord d'éviter le débat de fond sur le marasme provoqué par la volonté politique d'assurer l'accès généralisé à l'enseignement supérieur. Comme l'écrit Richard Münch à propos des universités de la fin du $\mathrm{XX}^{\mathrm{e}}$ siècle:

19 A l'exception de la petite minorité d'éléments motivés et prometteurs qui émergent de chaque cohorte et dont on garde un souvenir réconfortant, d'autant plus précieux qu'il est exceptionnel.

A ne pas confondre avec des évaluations de départements universitaires, fondées sur des procédures qualitatives, assimilables aux mécanismes de peer review adoptés par les publications scientifiques, et qui - sous certaines conditions - pourraient favoriser l'autorégulation des communautés scientifiques.

21 Contrairement à Berthoud et Busino (1995, p.10), je n'estime pas que Bloom soit excessif dans sa critique.

22 Peut-être faut-il préciser ici que l'auteur de ces lignes n'a pas eu à se plaindre jusqu'ici des résultats des évaluations fournies par ses étudiants. Il a toutefois le sentiment d'avoir souvent fait l'objet de jugements favorables pour de mauvaises raisons. 
«Auf die Offnung für die Massen folgt jetzt ihre Pädagogisierung und Umwandlung in reine Lehranstalten» (ibid., p. 150). Il s'agit ensuite de la compétition entre plusieurs modèles normatifs de la fonction d'enseignant: le magister, le pédagogue et l'animateur (Hirschhorn, 1993, pp. 230-239). Le modèle du magister, particulièrement adapté à l'université classique, est fondé sur une valeur centrale: «le savoir qui vaut en lui-même et pour lui-même». Il n'implique aucun refus de la pédagogie mais il suppose «que la pédagogie ne s'autonomise pas » (ibid., pp. 230-231). Le modèle du pédagogue, qui s'accorde mal avec la spécificité de l'institution universitaire, «ne fait plus du savoir la valeur centrale... Il y substitue l'enseigné, élève ou même étudiant.» (ibid., p. 233; souligné par JCH). Enfin, le modèle de l'animateur, «importé du milieu de l'entreprise » est centré sur un établissement d'enseignement particulier et exige une participation active à son fonctionnement et à son développement (ibid., pp. 235-238). Ce dernier modèle, combiné à celui du pédagogue, acquiert de nos jours une force normative peu commune, servant les intérêts de ceux qui ne brillent guère par leurs exploits intellectuels et plaçant injustement en position défensive le magister dont l'enseignement est lié à des activités de recherche lui offrant un autre cadre de référence, souvent bien plus stimulant. Il appartient également aux sociologues de montrer que la priorité accordée aux talents de communication et de gestion fait resurgir le malentendu fondamental sur la nature même de l'institution universitaire. En tant que foyer de création culturelle, l'université se doit de privilégier les contacts entre pairs, au-delà de barrières disciplinaires étroitement définies, entretenant ainsi un climat permanent d'émulation intellectuelle; c'est ce qu'avaient compris les fondateurs du groupe dit «de Montheron» tout en ayant, semble-t-il, le sentiment de mener un combat d'arrière-garde (1992). Lorsqu'un tel climat fait défaut, nul «atelier de pédagogie» ne peut prétendre sauver la mise. En revanche, là où une vie intellectuelle active est préservée, les étudiants, assistants et jeunes chercheurs en ressentent les effets bénéfiques sous forme d'effets de socialisation secondaire, comme tous les jeunes placés en situation d'apprentissage.

La même logique systémique simplificatrice qui affecte, on l'a vu, l'appréciation des tâches d'enseignement de l'université, sévit en matière de recherche et d'activité intellectuelle. La mise au point d'indicateurs de performance quantifiables - et jugés objectifs parce que quantifiables - est poussée jusqu'à l'absurde. Ainsi en est-il des «scores bibliométriques» dont tout universitaire quelque peu lucide connaît la signification ambiguë. Mais ce qui frappe surtout est la prégnance du modèle des sciences de la nature dans l'examen des projets de recherche, des demandes de bourses ou des rapports d'activité. Des critères d'évaluation dont on comprend bien l'intérêt pour des physiciens ou des biochimistes sont appliqués de façon irréfléchie aux praticiens des sciences humaines pour lesquels ils sont parfois totalement dénués de pertinence. Ainsi en est-il de l'usage généralisé de la langue anglaise ${ }^{23}$, de la mise en réseau, des publications dites mixtes, c'est-à-dire cosignées par des chercheurs de pays différents, de l'orientation directe vers la pratique, de la notion de «masse critique» si peu en harmonie

23 Ici encore est-il peut-être bon d'indiquer, pour éviter tout malentendu, que l'auteur de ces lignes s'exprime volontiers en anglais, verbalement ou par écrit, et qu'il a imposé l'usage de l'anglais comme troisième langue dans la Revue suisse de sociologie alors qu'il en assumait la direction. 
avec le caractère souvent artisanal de la recherche en sciences humaines. De nos jours, un spécialiste portugais de l'histoire régionale, par exemple, se verrait défavorisé dans la course aux crédits de recherche, pour n'être pas institutionnellement «en réseau » avec quelque collègue californien dont il aurait par ailleurs lu les publications ${ }^{24}$. Les sociologues qui ont l'oreille des instances dispensatrices de subventions se sont bornés pendant longtemps à des interventions portant sur la répartition des crédits. Certains d'entre eux commencent timidement à remettre en question les critères d'évaluation qui leur sont appliqués et qu'on les invite à appliquer lorsqu'ils sont appelés à émettre des avis sur les travaux de leurs collègues. Il serait souhaitable qu'ils aillent plus loin dans la critique d'une idéologie technobureaucratique privilégiant systématiquement la grande dimension en tant que telle, encourageant des projets-mammouths - programmes prioritaires, pôles d'excellence, etc. - qui ne peuvent que porter tort à l'ensemble des sciences humaines en présupposant imprudemment que du volume des ressources engagées surgira nécessairement la qualité et l'originalité des résultats. Mais ce qui s'observe en ce moment, en Suisse comme ailleurs, c'est plutôt l'acceptation résignée d'une dangereuse démesure. Nul doute que ce rappel des vertus de l'artisanat intellectuel en sciences humaines fera sourire. Mais qu'on nous cite une œuvre fondatrice, apte à faire école et à inspirer des vocations, qui ne soit pas l'œuvre d'un individu ou d'un petit groupe de collègues unis par des liens solides et des valeurs communes!

Revenons à présent à notre question de départ: d'où vient qu'une discipline dont les représentants les plus éminents ont volontiers joué le rôle de prophète, de conseiller du prince ou de critique social, ait fourni relativement peu de défenseurs vigoureux et d'analystes incisifs de l'institution universitaire ${ }^{25}$ ? S'agit-il de peur, d'aveuglement ou de complicité? Un peu de tout cela, sans doute. Plus que d'autres disciplines relevant des sciences sociales et humaines, la sociologie se trouve en situation de symbiose avec l'université, ne disposant pas de débouchés professionnels comparables, par exemple, à ceux de l'histoire, de la géographie ou de la psychologie. Certes, dans les milieux de sociologues, on parle volontiers aujourd'hui «des métiers » de la sociologie et de nombreux diplômés en sociologie trouvent à s'employer dans des secteurs d'activité très divers. Mais leurs activités professionnelles n'ont souvent plus grand-chose à voir avec l'exercice de la sociologie proprement dite. Dans les cas les plus favorables, il s'agit de manier avec plus ou moins d'habileté quelques techniques de recherche que la sociologie partage avec d'autres disciplines, à des fins de comptabilité sociale ou de sondage de l'opinion, sans avoir pour autant la possibilité de faire appel aux catégories conceptuelles propres à l'analyse sociologique. En tant que projet scientifique original, l'entreprise sociologique ne doit donc sa survie qu'à la bienveillance des responsables de l'institution universitaire. Ceci est bien plus qu'une figure de style: aux Etats-Unis, aux Pays-Bas - où la sociologie connut un développement

24 L'exemple est fictif, mais il m'est directement inspiré par ma participation récente à une évaluation d'unités de recherche effectuée à la demande de la Fundação para a Ciência e a Tecnologia à Lisbonne.

25 En Suisse romande, François-Xavier Merrien est un des rares à aborder à cet égard des questions de fond et à ne pas craindre d'affronter l'opinion, comme en témoignent ses articles dans la presse régionale $(1996,1997)$. 
extraordinaire dans les années soixante ${ }^{26}$-, des départements de sociologie ont tout bonnement été fermés au cours des dernières décennies parce que leurs productions scientifiques avaient déçu ceux qui avaient conçu des attentes - peut-être irréalistes - à leur égard ${ }^{27}$. C'est dire que le système universitaire est un des supports majeurs de l'existence de la sociologie en tant que discipline. Dès lors, la crise de l'université est aussi le drame de la sociologie institutionnalisée, comme je l'ai déjà indiqué précédemment (1995b, p. 186). Peter Berger, auteur de la célèbre Invitation to Sociology, faisait observer il y a quelques années que rares étaient de nos jours les sociologues pratiquant une sociologie «dans la tradition des classiques, fondée sur la connaissance de l'histoire, méthodologiquement flexible, imprégnée d'un esprit cosmopolite, infiniment curieuse de chaque manifestation de la vie humaine» $(1994$, p. 6). Il ajoutait que «le système de formation et le système de gratifications de la profession» sont organisés de manière à empêcher l'émergence de telles personnes (ibid.). Comme pourrait-il en être autrement, sur ce grand navire en perdition qu'est l'université d'aujourd'hui? Le modèle hybride, mi-collégial mi-bureaucratique, vers lequel elle a évolué s'écarte de plus en plus de la concrétisation, même approchée, du forum de communication intellectuelle optimale pensé par Habermas ${ }^{28}$.

On en arrive ainsi à un véritable cercle vicieux organisationnel: l'institution universitaire assure en priorité la promotion des sociologues qui sont le moins à même d'enrayer son déclin. Il y a tout d'abord ceux qu'on peut à bon droit qualifier de complices du naufrage. Cette complicité est celle des «intellectuels-administrateurs », déjà raillés par Charles Wright Mills (1959, 1967, pp. 111 et ss.) et fustigés par Alvin Gouldner (1970, 1972, p. 504). Ils ont appris à se mouvoir avec aisance sur le pont qui donne de la bande. La dégradation de l'institution universitaire ne les affecte guère. Ils en profitent même dans la mesure où ils sont gagnés par l'ethos de «l'animateur» et bénéficient largement de la bureaucratisation de la recherche, mortelle pour leur vie intellectuelle mais pas pour leur appétit de pouvoir. Il y a ensuite ceux dont l'aveuglement est manifeste. Ils en sont arrivés à si bien s'identifier au navire en perdition qu'ils n'en perçoivent nullement la dérive. Nourris dans le sérail, ils en connaissent tous les détours mais ne se passionnent que pour des manœuvres à courte vue que n'inspire plus aucune préoccupation authentiquement intellectuelle. Ceux-là sont tout aussi loin du profil esquissé par Peter Berger mais ils sont en revanche les virtuoses de l'intrigue et de l'astuce. Leur habileté est indéniable et peut avoir quelque chose de rassurant dans l'immédiat. Par leurs attitudes et leur comportement, ils suggèrent en effet que tout problème trouvera toujours une solution dans le bricolage administratif et l'improvisation au jour le jour. En fait, leur rouerie même les empêche de prendre toute la mesure de la catastrophe institutionnelle qu'ils sont en train de vivre.

26 Développement qui ne fut perçu qu'une vingtaine d'années plus tard dans les milieux de langue française (cf. à ce sujet: Chenal, 1983), à un moment où le déclin commençait à s'amorcer.

27 Pour ce qui est des Etats-Unis, voir le livre de Irving Horowitz, The Decomposition of Sociology dont l'auteur écrit «It is more a matter of pain than pride to have felt the need to write such a book» (1994, p. 3).

28 Voir à ce sujet mon évocation d'un département universitaire de fiction où la dissimulation serait devenue l'arme la plus classique de l'action stratégique ( Coenen-Huther, 2000, pp. 143-144). 
Et puis la crainte est là, secrète, inavouée, diffuse. A l'heure où «le poids de l'économie ou des politiques publiques est déterminant» (Busino, 1999, p. 125), l'angoisse du lendemain a souvent quelque chose de paralysant. L'obsession des coupures budgétaires et des suppressions de postes hante les esprits. L'inquiétude face à la précarisation menaçante à terme est très réelle chez ceux qui ne sentent que trop bien la fragilité d'une légitimité scientifique mesurée selon les normes de rendement à la mode; voués à une tâche d'élucidation, les sociologues ne seront jamais les dentistes ou les chirurgiens du social. Mais cette crainte, tout à fait fondée, ne se double que rarement d'une prise de conscience réelle de la gravité des enjeux. Les intéressés ne semblent pas se rendre compte que leur sort est indissolublement lié au renouveau de l'université classique, refuge des disciplines qui ne peuvent se prévaloir d'une utilité pratique directe. Et le fait qu'il n'y ait pas, au sein de la corporation des sociologues, de ralliement autour de valeurs communes pour la défense de l'Alma Mater reflète bien la situation d'une discipline pluriparadigmatique, fragmentée à l'extrême. Si quelque chose unit encore les membres influents de cette «communauté sociologique éclatée» (Busino, 1993, pp. 9-10), c'est la peur d'aller à contre-courant et d'affronter la sensibilité égalitariste dominante alors que la dévalorisation des diplômes universitaires ne profite bien évidemment à personne. Dans ces conditions, les stratégies d'adaptation sont individuelles: elles sont de plus en plus fréquemment le ritualisme et l'évasion (retreatism) au sens que Merton a donné à ces termes (1968, pp. 203-209). Collectivement, les sociologues renoncent ainsi à situer le marasme universitaire dans un contexte sociétal marqué par une crise renouvelée du lien social, par l'obsolescence rapide des connaissances et par la faillite de la transmission intergénérationnelle de culture. Ils se privent ainsi de la possibilité de proposer un aggiornamento crédible de l'université en jouant de leur atout majeur qui est la référence à la totalité sociale.

Département de sociologie

Université de Genève

\section{BIBLIOGRAPHIE}

Aron, Raymond, La Révolution introuvable, Paris, Fayard, 1968.

Berger, Peter L., Does Sociology Still Make Sense?, in «Schweizerische Zeitschrift für SoziologieRevue Suisse de Sociologie», Vol. 20, No 1, 1994, pp. 3-12.

Berthelot, Jean-Michel, Projet universitaire et dynamique de l'évaluation, in «L'Université en question », Université de Toulouse-Le Mirail, 1990, pp. 15-19.

Berthoud, Gérald et Busino, Giovanni, Pratiques sociales et théories. Les discordes des universitaires, Genève, Droz, 1995.

Bienaymé, Alain, L'enseignement supérieur et l'idée d' université, Paris, Economica, 1986.

Bloom, Allan, The Closing of the American Mind, New York, Simon and Schuster, 1987; tr. fr. L' âme désarmée, Paris, Julliard, 1987.

Boudon, Raymond, Effets pervers et ordre social, Paris, Presses Universitaires de France, 1979.

Boudon, Raymond, Les intellectuels et le second marché, in «Revue européenne des sciences sociales », No 87, 1990, pp. 89-103.

Bourdieu, Pierre, Homo Academicus, Paris, Minuit, 1984. 
Busino, Giovanni, Critiques du savoir sociologique, Paris, Presses Universitaires de France, Coll. «Sociologies», 1993.

Busino, Giovanni, Plaidoyer pour l'Université, in: Busino, G., Honorez, I. et Miéville, A., « Notes et documents pour une histoire et une sociologie des prix Nobel scientifiques suisses », Université de Lausanne, IAS, 1999, pp. 115-129.

Chenal, Odile, Les sociologues et les institutions : le cas des Pays-Bas, in "L'Année sociologique», Vol. 33, 1983, pp. 37-65.

Coenen-Huther, Jacques, Competing Rationalities : the Students and their Studies, in «Social Science Information », Sage, Londres, Vol. 28, No 4, 1989, pp. 769-773.

Coenen-Huther, Jacques, Observation participante et théorie sociologique, Paris, L'Harmattan, 1995a.

Coenen-Huther, Jacques, Crise de la sociologie ou sociologues en crise?, in «Revue européenne des sciences sociales », Tome XXXIII, No 100, 1995b, pp. 185-189.

Coenen-Huther, Jacques, The Paths of Recognition : Boudon, Bourdieu and the «Second Market» of Intellectuals, in «International Journal of Contemporary Sociology», Vol. 35, No 2, 1998, pp. 208-216.

Coenen-Huther, Jacques, Stratégies de dissimulation et rationalité limitée, in: André Petitat, Ed., «Secret et lien social», Paris, L'Harmattan, 2000, pp. 137-146.

Cohen, Michael D., March, James G. et Olsen, Johan P., A Garbage Can Model of Organizational Choice, in «Administrative Science Quarterly», XVII, 1972, pp. 1-25.

Cohen, Michael D., March, James G. et Olsen, Johan P., People, Problems, Solutions and the Ambiguity of Relevance, in: March, James G. et Olsen, Johan P., Eds, 1976, pp. 24-37.

Endruweit, Günter, Die Universitätsidee in den Zeit des Sparzwangs, in «Politische Studien», Heft 352, 48. Jahrgang, März-April 1997, pp. 147-155.

Finkielkraut, Alain, La révolution cuculturelle à l'école, in «Le Monde», 19 mai 2000, pp. 1 et 19.

Fitoussi, Jean-Paul, et Rosanvallon, Pierre, Le nouvel âge des inégalités, Paris, Seuil, 1996.

Friedberg, Erhard, Le pouvoir et la règle. Dynamiques de l'action organisée, Paris, Seuil, 1993.

Friedberg, Erhard et Musselin, Christine, En quête d'universités. Etude comparée des universités en France et en RFA, Paris, L'Harmattan, 1989.

Gouldner, Alvin W., The Coming Crisis of Western Sociology, 1970, Réimpression Heinemann, HEB Paperback, 1972.

Groupe de Montheron, Des hommes de science aux prises avec le temps, Lausanne, Presses polytechniques et universitaires romandes, 1992.

Hirschhorn, Monique, L'ère des enseignants, Paris, Presses Universitaires de France, 1993.

Horowitz, Irving L., The Decomposition of Sociology, New York, Oxford University Press, 1994.

Kleiber, Charles, Pour l' Université. Histoire des lieux et enjeux. L'Université de demain. Opinions et débats. S.l.n.d.

Lasch, Christopher, The Culture of Narcissism. American Life in an Age of Diminishing Expectations, New York, Norton and Company, 1979.

Le Monde, Les nouveaux publics étudiants ébranlent les premiers cycles universitaires, 12 mai 2000, p. 9.

Lepoutre, David, Certains collègues craignent les jeunes de banlieue. Propos recueillis par Stéphanie Le Bars, in «Le Monde», 12 mai 2000, p. 9.

Lodge, David, Nice Work, roman, Harmondsworth, Penguin Books, 1989.

March, James G. et Olsen, Johan P., Eds, Ambiguity and Choice in Organizations, Bergen, Universitetsforlaget, 1976.

March, James G. et Simon, Herbert A., Organizations, New York, Wiley, 1958; tr. fr. Les organisations, Paris, Dunod, 1964.

Mathews, Jay et Argetsinger, Amy, To Raise Test Scores, School Pressure Teachers to Cheat, in «International Herald Tribune», June 3-4, 2000, p. 3. 
Merton, Robert K., Social Theory and Social Structure, Enlarged edition, New York, The Free Press, 1968.

Merrien, François-Xavier, L'avenir de la Suisse dépend aussi de sa politique universitaire, in «Le Nouveau Quotidien», 22 et 23 octobre 1996.

Merrien, François-Xavier, Université : la part des étrangers, in « Journal de Genève et Gazette de Lausanne», 18 mars 1997.

Mills, C. Wright, The Sociological Imagination, New York, Oxford University Press, 1959; tr. fr. L'imagination sociologique, Paris, Maspero, 1967.

Morin, Edgar, La tête bien faite, Paris, Seuil, 1999.

Münch, Richard, Editorial, in «Soziologische Revue», Vol. 21, No 2, 1998.

Musselin, Christine, Structures formelles et capacités d' intégration dans les universités françaises et allemandes, in «Revue française de sociologie», XXXI/3, 1990, pp. 439-461.

Rüegg, Walter, Der Mythos der Humboldtschen Universität, in «Universitas in theologia - theologia in universitate». Festschrift für Hans Heinrich Schmid zum 60. Geburtstag, TVZ, Zürich.

Weick, K. E., Educational Organizations as Loosely Coupled Systems, in «Administrative Science Quarterly», XXI, 1976, pp. 1-19.

Zijderveld, Anton C., Staccato cultuur, flexibele maatschappij en verzorgende staat [Culture «staccato », société flexible et Etat protecteur], Utrecht, Lemma, 1991. 